\title{
Designing Auditory Feedback from Wearable Weightlifting Devices
}

\author{
Mengyue Pan \\ School of Informatics and Computing \\ Indiana University \\ Indianapolis, IN, USA \\ zoepan@umail.iu.edu

\section{Sagar Salvi} \\ School of Informatics and Computing \\ Indiana University \\ Indianapolis, IN, USA \\ ssalvis@umail.iu.edu

\section{Erin Brady} \\ School of Informatics and Computing \\ Indiana University \\ Indianapolis, IN, USA \\ brady@iupui.edu
}

Permission to make digital or hard copies of part or all of this work for personal or classroom use is granted without fee provided that copies are not made or distributed for profit or commercial advantage and that copies bear this notice and the full citation on the first page. Copyrights for third-party components of this work must be honored. For all other uses, contact the Owner/Author.

CHI'18 Extended Abstracts, April 21-26, 2018, Montreal, QC, Canada (C) 2018 Copyright is held by the owner/author(s)

https://doi.org/10.1145/3170427.3188692

\begin{abstract}
While wearable devices for fitness have gained broad popularity, most are focused on tracking general activity types rather than correcting exercise forms, which is extremely important for weightlifters. We interviewed 7 frequent gym-goers about their opinions and expectations for feedback from wearable devices for weightlifting. We describe their desired feedback, and how their expectations and concerns could be balanced in future wearable fitness technologies.
\end{abstract}

\section{Author Keywords}

Weightlifting; wearable device; exercise; feedback.

\section{ACM Classification Keywords}

H.5.2. Information interfaces and presentation (e.g., $\mathrm{HCI}$ ): User Interfaces.

\section{Introduction}

People often go to the gym with clear goals - to lose weight, gain strength, or build body tone. To achieve these goals, they may use exercise machines which target specific muscle groups - but these machines require precise movements which may be hard for newcomers to learn properly and avoid injury. Even elite weightlifters reported having acute or chronic injuries due to performing weightlifting incorrectly [2]. 


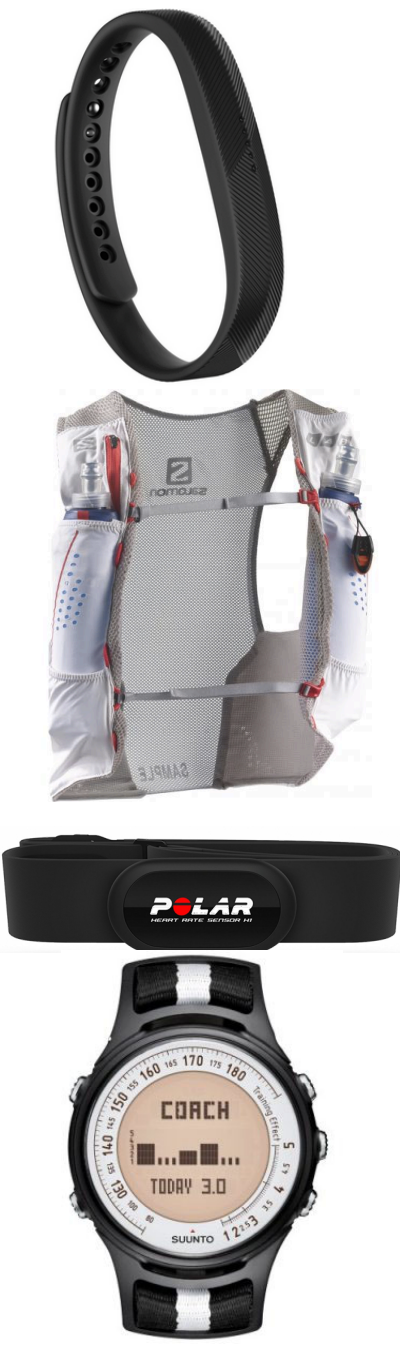

Figure 1: Commercial fitness tracking devices (top to bottom: FitBit, SenseVest, Polar Heartrate, and Sunnto T4).
Wearable devices have been designed to help exercisers track daily activity levels and correct their exercise forms. However, most academic work in this area has focused improving the precision of activity recognition for these exercises $[4,7]$. There has not been detailed research on the types of feedback these devices could offer users that would lead to better workout experiences maximized exercise efficiency.

In this late-breaking work, we describe a study of 7 active gym users' reactions to videos of the Ollinfit, a commercial wearable device that corrects weightlifting forms. Brainstorming with our participants resulted in expected features of workout wearables and suggestions to improve the feedback of these devices. For example, the feedback could provide tailored, motivating information like a personal trainer does to inspire the lifter; or could provide users with analytical data to inform them about their exercise trends and predictions. These findings have greater implications for any real-time, precise feedback on task performance.

\section{Related Work}

Many devices exist to measure calorie burn rates, steps taken, body temperature, heart rate, or performance while exercising (Figure 1). While the wrist-worn FitBit device is best known publicly, other devices also collect data and provide feedback to assess the quality of physical activity. The SenseVest is a sensor integrated into a shirt, that records a user's body temperature and heartrate and sends the data to a remote computer for them to analyze. The Polar Heartrate monitor combines a wrist-worn and chest-worn unit which monitors users' heart rate and calorie burns, giving aural feedback once calorie burn levels reach a certain amount. The Sunnto T4 monitors physical parameters such as heart rate, burned calories, speed and distance, and makes suggestions for adjustments in the user's workout routine (along with an estimation on how the workout improves user's aerobic fitness).

In academic research, systems have been developed to encourage healthy exercise routines. Buttussi et al. introduced MOPET [1], a system of wearable devices and a virtual trainer to help runners navigate a route, get real-time feedback and suggest physical activities for users while exercising outdoors. Other tools were designed to motivate physical activity, such as UbiFit Garden [3], which recognized physical activity with a mobile phone and an on-body sensor. The range of activities that could be inferred by the system was large, but heavily relied on physical location for context and could not detect specific activity-level mistakes.

Other projects explored detecting specific activities precisely using wearable devices. Those works mainly focused on detecting mistakes from the exercise and activity recognition. Kowsar et al. [4] designed a system that could detect deviation from correct weightlifting performance. Velloso et al. [6] used a modelbased system which could adjust its parameters for different users and activities to detect mistakes during weight-lifting and improve performance. Those systems mainly focused on activity recognition and mistake detection. Other work focused on feedback given to users while using wearable devices to do exercise.

Rector et al. [5] designed eyes-free yoga to help blind or low-vision individuals to learn yoga poses with the audio instructor for 6 standing yoga poses and got useful and customized feedbacks for future work. In this paper, we focus on feedback to provide better instructions for users of wearable fitness devices. 


\section{Methods}

Study Design

The Ollinfit ${ }^{1}$ is an in-development weightlifting aid that combines a mobile app and 3 wearable sensors on the chest, legs, and wrist. The sensors provide vibrate feedback to help users correct their form, provide postworkout details about technique, and records weightlifting process. Since this device is not yet commercially available, we showed our participants a video of the device to gauge their impressions.

We conducted interviews with frequent gym users about their experiences with wearable devices, reactions to the video of the Ollinfit device, desired features for wearable devices, and problems within the devices. Participants were compensated with a $\$ 20$ digital gift card. This study was approved by our institution's IRB, and interviews lasted 15-30 minutes.

\section{Recruiting}

We recruited participants with exercise experience in collaboration with the National Institute for Fitness and Sport (NIFS). We set up a table in the entrance of the facility and recruited 4 participants. We additionally sent messages to our contacts on social media platforms to recruit participants we knew were frequent gym users. 3 participants were recruited directly.

\section{Participants}

We recruited 7 participants who had experience of weight-lifting in our study ( 5 female, 2 male). Their average age was 23.8 years with a range between 21 to 27 years. All had exercised frequently for at least 2 years, and all went to gym between 3-7 days a week.

${ }^{1}$ http://www.ollinfit.com
All participants performed weightlifting as part of their exercise routine, but some of them engaged in other forms of exercise, such as cardio activities or group courses like Zumba.

Due to NIFS's proximity to the IUPUI campus, all of our participants were students (4 undergrads, 1 Masters student, and $2 \mathrm{PhD}$ students). Three of the undergraduate students were in majors related to physical activity, where they have learned specific fitness techniques. 2 participants learned the right way of doing weightlifting either from certificated personal trainers, and another was trained by a friend with personal training experience.

\section{Responses to Existing Workout Technology}

Below we describe our participants' experiences with wearable exercise devices. Then we discuss their suggestions for enjoyable and customized feedback for future development of workout technologies.

Experience with Technologies

We explored participants' experience on exercise devices before showing them the video of the Ollinfit. Two participants had not used any exercise technologies at the gym before. The other participants had used a mix of manual or automatic recording technologies to track their fitness practices.

One participant had used MyFitnessPal, a free smartphone app, to record calorie input, manage their diet, and plan workout sessions. This information allowed them to accurately evaluate how many calories they burned from daily life activities or from working out, and be aware of the calories they consumed, which helped them to lose weight or gain muscle. 


\section{Quote 1:}

"I would really use my phone for - sometimes I just record or just do notes like how much I lifted, so I could try to lift a little bit more the next week or something". -P5

\section{Quote 2:}

"I like the idea that it has the feedback to actually correct you a little bit because the video we have right now, it's just 'follow the other movements', so there's no way for them to tell you if the gestures are incorrect". $-P 2$

\section{Quote 3:}

"So if you do a core

exercise, it involves lots of muscle movements. Where I should put the sensors on? How can it monitor other muscles?" -P.

Quote 4:

"...if it can load programs, then I definitely can learn something I've never done before, but if it can't, it still can be helpful... it can help me to correct my, you

know, my movements" $-P 3$
Another participant manually recorded the activities they did at the gym by taking notes on their phone without a specific fitness-oriented application. This participant used their occasional notes as motivation for future workouts (Quote 1, in the sidebar).

2 participants had experience using a Fitbit to record data either during exercise or outside of the gym. Another participant used a wearable device on her chest to record performance during her exercise classes, so she could access data after her classes.

In addition to traditional resources for learning how to correctly perform workout activities, all of our participants noted that they also learned exercise forms from watching videos on YouTube. One participant additionally used Instagram, a photo and video-based social media platform, to learn how to perform different exercises. These responses indicate that online social platforms can be a resource for gym users to be introduced to movements or poses, but do not provide any opportunity for real-time or post-workout feedback on the correctness of moves performed.

\section{Reaction to the Ollinfit}

All 7 participants thought the Ollinfit was an interesting or helpful device, especially since it could correct users' poses through audio feedback (Quote 2). Our respondents felt that with the Ollinfit, they could correct their movements by themselves and exercise effectively with verbal corrections.

Some participants asked how the device works, curious to know how accurate the device could be because of the diversity of individuals and exercises. Due to the difference of body structure between individuals, 1 participants noted whether the device could monitor all of the users' movements and gave right directions according to their body structure. Another participant mentioned whether the device could monitor the whole body, since it only has 3 sensors, she doubted the device could detect all the muscle movements across the whole body in order to provide precise feedbacks (Quote 3).

1 participant asked whether the device could upload new programs and the device could monitor new movements. There are multiple new movements developed overtime, some new movements are the combination of basic poses, and some movements are created with new forms. The participant hoped that she could upload new forms and the device could detect new forms and correct her (Quote 4).

\section{Expectations for Future Workout Technology}

Based on their prior experiences with workout

technologies, and their exposure to the Ollinfit device, we asked participants to ideate about potential features and challenges of workout devices that would help them in their exercise routines.

Desired Features of Future Devices

Positive FeEdBACK: Feedback is important for users to correct their movements, and positive feedback can motivate users and direct them in a good way to exercise. The Ollinfit corrects users' movements by telling users what to do, sometimes even repeating phrases several times which makes users feel upset. This response may reveal that gym-goers are highly sensitive to potential judgement by workout technologies, and felt that negative or even neutral 


\section{Quote 5:}

"Instead of telling me "it's not right, it's not right" because I know I'm not right, but if I'm doing something better than before, than the previous one, it should tell me, and I don't need it to tell me all the time". $-P 2$

\section{Quote 6:}

"...not something that is designed for everyone - I want some customized and personalized design that can learn my habits, it learns my schedule, it learns my eating and everything and generates exercise plan just for myself". $-P 2$

\section{Quote 7:}

"The only [problem] I could forsee is that some people when they are in the gym they get embarrassed when some people tell them how to do something. So if you could have that go through headphones or something to tell people, to tell them correct it or something." $-P 5$ feedback might discourage them from using a fitness device (Quote 5).

PERSONALIZED DESIGN: Individuals have different goals during exercise (such as lose weight, gain muscle, train glutes), diverse eating habit. It would be perfect to maximize work-out results by learning users' eating, training habits and generating personalized plans for them (Quote 6).

UPLOAD NEW WORKOUTS: Based on our interviews, all of our participants learned exercise forms from YouTube or other social media like Instagram. Those media could only show them correct movements, but does not include the ability to correct mistakes. Due to the performance diversity of weightlifting, there are always new movements been created or combined with several other forms. So, if the users could upload new forms they learned from social media or somewhere else to the device, it would maximize their exercise efficiency.

HEADPHONES: The Ollinfit did not show headphone compatibility for users. As the device gives feedback, others around the user could hear the voice which may make the users feel embarrassed (Quote 7)

\section{Concerns about Workout Devices}

Despite the potential of these devices, users listed a number of potential concerns that might arise.

PHYSICAL INJURY: The Ollinfit corrects users' movements through audio feedback. Since the Ollinfit was mainly designed for weight-lifting, stimulating targeted muscle could maximize work-out results. Hence, some users may exercise with heavy loads in order to stimulate their muscles. But the Ollinfit cannot not detect each users' maximum and minimum endurance of loads - as users try to perform at a higher level of weight, injury might occur that could not be prevented.

DISTRACTION: One participant noted that auditory feedback could distract him depending on how many times and when the system gave feedback. If the device starts to give feedback loudly and abruptly when users are focused on training, it might startle the users, and repetition may distract users from training.

\section{Discussion}

Our participants' responses revealed a number of important values and concerns for workout technology design, such as concerns over being judged at the gym and a desire for a realistic training experiences. Below we provide initial ideas on how these values could be incorporated into new corrective workout technologies.

ReAL-TIME FEedBACK: Real-time feedback should be positive and motivating, similar to the feedback provided by human physical trainers. The repetitive robotic feedback could be replaced by a real human voice with emotional and positive feedback, such as "you're doing better this time, keep going". This feedback should be dynamic, such as incrementa feedback according to the performance of users starting with very short instruction on how to correct a movement; but increasing feedback if the error continues. Feedback could also teach users by giving reasoning or what kind of injury could be caused if the error continues.

POST-SET FEEDBACK: Weightlifting combines short periods of physical motion with breaks between sets of exercise repetitions. These breaks provide a valuable 
opportunity to offer this additional information, like reasoning behind workouts or specific goals, while addressing concerns about privacy and distraction. During the breaks, the device could offer some knowledge via video, which users could observe for a short period through headphones.

POST-WORKOUT FEEDBACK: The device should record how many sets, reps, and weights were used each time to allow for in-the-moment workout adjustment. However, this data is also useful for long-term fitness

improvement and reflective analysis. Analyzing the data could motivate users by highlighting

improvements, such as showing improvements on the home page. This data also lends itself to generating predictions and trends, such as offering users with graphs of their ideal versus realistic calorie burns or monitoring changes in exercise patterns and offering future workouts suggestions accordingly.

\section{Future Work}

We plan to implement and test our users' suggestions via a Wizard of Oz prototype. Our work could extend to other demographics - Rector et al. [5] note that blind and low-vision individuals tend to perform less physical activity than sighted peers, which can cause problems such as obesity or mental health. We hope to design a device which could help people with visual impairments to do weightlifting in the gym or at home to help them be more active and reduce health problems.

\section{References}

1. Fabio Buttussi, Luca Chittaro, and Daniele Nadalutti. 2006. Bringing mobile guides and fitness activities together: a solution based on an embodied virtual trainer. In Proceedings of the 8th conference on
Human-computer interaction with mobile devices and services, 29-36.

2. Gregg Calhoon and Andrew C Fry. 1999. Injury rates and profiles of elite competitive weightlifters. Journal of athletic training 34, 3: 232.

3. Sunny Consolvo, David W. McDonald, Tammy Toscos, Mike Y. Chen, Jon Froehlich, Beverly Harrison, Predrag Klasnja, Anthony LaMarca, Louis LeGrand, and Ryan Libby. 2008. Activity sensing in the wild: a field trial of ubifit garden. In Proceedings of the SIGCHI conference on human factors in computing systems, 1797-1806.

4. Yousef Kowsar, Masud Moshtaghi, Eduardo Velloso, Lars Kulik, and Christopher Leckie. 2016. Detecting unseen anomalies in weight training exercises. In Proceedings of the 28th Australian Conference on Computer-Human Interaction, 517-526.

5. Kyle Rector, Cynthia L. Bennett, and Julie A. Kientz. 2013. Eyes-free yoga: an exergame using depth cameras for blind \& low vision exercise. In Proceedings of the 15th International ACM SIGACCESS Conference on Computers and Accessibility, 12.

6. Eduardo Velloso, Andreas Bulling, Hans Gellersen, Wallace Ugulino, and Hugo Fuks. 2013. Qualitative activity recognition of weight lifting exercises. In Proceedings of the 4th Augmented Human International Conference, 116-123.

7. Darragh Whelan, Martin O'Reilly, Tomás E. Ward, Eamonn Delahunt, and Brian Caulfield. 2016.

Evaluating performance of the lunge exercise with multiple and individual inertial measurement units. In Proceedings of the 10th EAI International Conference on Pervasive Computing Technologies for Healthcare, 101-108. 